\title{
Distribution of $\beta$-carotene-encapsulated polysorbate 80-coated poly(D, L-lactide-co-glycolide) nanoparticles in rodent tissues following intravenous administration
}

This article was published in the following Dove Press journal:

International Journal of Nanomedicine

27 November 2015

Number of times this article has been viewed

\author{
Taiki Miyazawa ${ }^{1,2}$ \\ Kiyotaka Nakagawa ${ }^{1,2}$ \\ Takahiro Harigae ${ }^{2}$ \\ Ryo Onuma ${ }^{2}$ \\ Fumiko Kimura ${ }^{2}$ \\ Tomoyuki Fujii ${ }^{3}$ \\ Teruo Miyazawa ${ }^{4,5}$ \\ 'Vascular Biology Laboratory, \\ Jean Mayer USDA (United States \\ Department of Agriculture)-Human \\ Nutrition Research Center on Aging, \\ Tufts University, Boston, MA, USA; \\ ${ }^{2}$ Food and Biodynamic Chemistry \\ Laboratory, ${ }^{3}$ Terahertz Optical \& Food \\ Engineering Laboratory, Graduate \\ School of Agricultural Science, ${ }^{4}$ Food \\ and Biotechnology Innovation Project, \\ New Industry Creation Hatchery \\ Center (NICHe), ${ }^{5}$ Food and Health \\ Science Research Unit, Graduate \\ School of Agricultural Science, Tohoku \\ University, Sendai, Japan
}

Correspondence: Kiyotaka Nakagawa Food and Biodynamic Chemistry Laboratory, Graduate School of Agricultural Science, Tohoku University, I-I Tsutsumidori-Amamiyamachi, Aobaku, Sendai 98I-8555, Japan

Tel +8I 227178906

Fax +8I 227178905

Email nkgw@m.tohoku.ac.jp
Purpose: Biodegradable nanoparticles (NPs) composed of poly(D, L-lactide-co-glycolide) (PLGA) have attracted considerable attention as delivery systems of drugs and antioxidative compounds, such as $\beta$-carotene (BC). Intravenous (IV) administration of BC-containing PLGANPs (BC-PLGA-NPs) coated with polysorbate 80 (PS80) has been shown to effectively deliver $\mathrm{BC}$ to the brain. However, the whole-body distribution profile of $\mathrm{BC}$ is still not clear. Therefore, we investigated the accumulation of $\mathrm{BC}$ in various organs, including the brain, following IV administration of PS80-coated BC-PLGA-NPs in rats.

Methods: PS80-coated and uncoated BC-PLGA-NPs were prepared by solvent evaporation, and administered intravenously to Sprague Dawley rats at a BC dose of $8.5 \mathrm{mg} /$ rat. Accumulation of BC in various organs (brain, heart, liver, lungs, and spleen) and blood plasma was evaluated by high performance liquid chromatography with ultraviolet (UV) detection, 1 hour after administration. Results: We prepared PS80-coated BC-PLGA-NPs with an entrapment efficiency of 14\%, a particle size of $260 \mathrm{~nm}$, and a zeta potential of $-26 \mathrm{mV}$. Coating with PS80 was found to result in significant accumulation of $\mathrm{BC}$ in the lungs, rather than in the brain and other tissues. Further, plasma levels of BC in the PS80-coated BC-PLGA-NP group were much lower than those of the uncoated BC-PLGA-NP group.

Conclusion: Following IV administration, PS80-coated BC-PLGA-NPs are quickly transferred from plasma circulation to the lungs, rather than the brain. Significant accumulation of BC in the lungs may be useful for health-related applications.

Keywords: $\beta$-carotene, intravenous administration, nanoparticles, poly(D, L-lactide-coglycolide) (PLGA), polysorbate 80 (PS80), tissue distribution

\section{Introduction}

Use of biodegradable polymer-based nanoparticles (NPs) for drug delivery purposes can result in enhanced distribution of drugs to target organs. ${ }^{1}$ One of the most attractive biodegradable polymers used for the preparation of NPs is poly $(\mathrm{D}, \mathrm{L}-$ lactide-coglycolide) (PLGA). ${ }^{2}$ PLGA undergoes hydrolysis in the body to produce the naturally occurring monomers lactic acid and glycolic acid. ${ }^{3}$

Intravenous (IV) administration of PLGA-based NPs (PLGA-NPs) coated with nonionic surfactants has been shown to result in accumulation in specific tissues. ${ }^{4}$ For example, IV administration of polysorbate 80 (PS80)-coated PLGA-NPs results in significant uptake by the brain. ${ }^{5-7}$ However, to the best of our knowledge, few studies have investigated the effect of PS80 coating on whole-body tissue distribution of NPs. 
Recently, PLGA-NPs have been shown to be useful as delivery systems of the bioactive compound $\beta$-carotene $(\mathrm{BC}$, Figure 1A) for inhibition of oxidative stress within organs. ${ }^{8}$ However, there is currently no data available regarding the tissue distribution of IV-administrated PS80-coated BCencapsulated PLGA-NPs (BC-PLGA-NPs), although one study did report that intraperitoneal (IP) administration of PS80-coated BC-PLGA-NPs resulted in a significant accumulation of $\mathrm{BC}$ in the brains of rats. ${ }^{9}$

Herein, we prepared PS80-coated and uncoated BC-PLGA- NPs by solvent evaporation, and evaluated the surface morphology and BC entrapment efficiency (EE) of the NPs by scanning electron microscope (SEM) and high performance liquid chromatography with UV detection (HPLC-UV), respectively. Mean NP diameters and zeta potentials were measured by dynamic light scattering (DLS) and laser Doppler anemometry, respectively. PS80-coated BC-PLGA-NPs, uncoated BC-PLGA-NPs, and saline control were administered intravenously to Sprague Dawley rats, and the corresponding tissue distribution profiles of
BC, including brain uptake, were evaluated by HPLC-UV (Figure 1B).

\section{Materials and methods Materials}

BC powder (purity $>95 \%$ ) was purchased from Wako Pure Chemical Industries, Ltd. (Osaka, Japan). Membrane filters (Millex-HV, $0.45 \mu \mathrm{m}, 33 \mathrm{~mm}$ ) were obtained from EMD Millipore (Billerica, MA, USA). PLGA (50:50, molecular weight $=30,000-60,000)$ and poly vinyl alcohol (PVA, molecular weight $=30,000-70,000,87 \%-90 \%$ hydrolyzed) were obtained from Sigma-Aldrich (St Louis, MO, USA). PS80 was purchased from Nihon Yushi Co., Ltd. (Tokyo, Japan). All chemicals and reagents used were of analytical grade or higher.

\section{Preparation of BC-PLGA-NPs: surface morphology and EE analysis}

BC-PLGA-NPs were prepared by solvent evaporation. ${ }^{10,11}$ Briefly, $20 \mathrm{mg}$ of free (commercial) BC powder and $200 \mathrm{mg}$ of

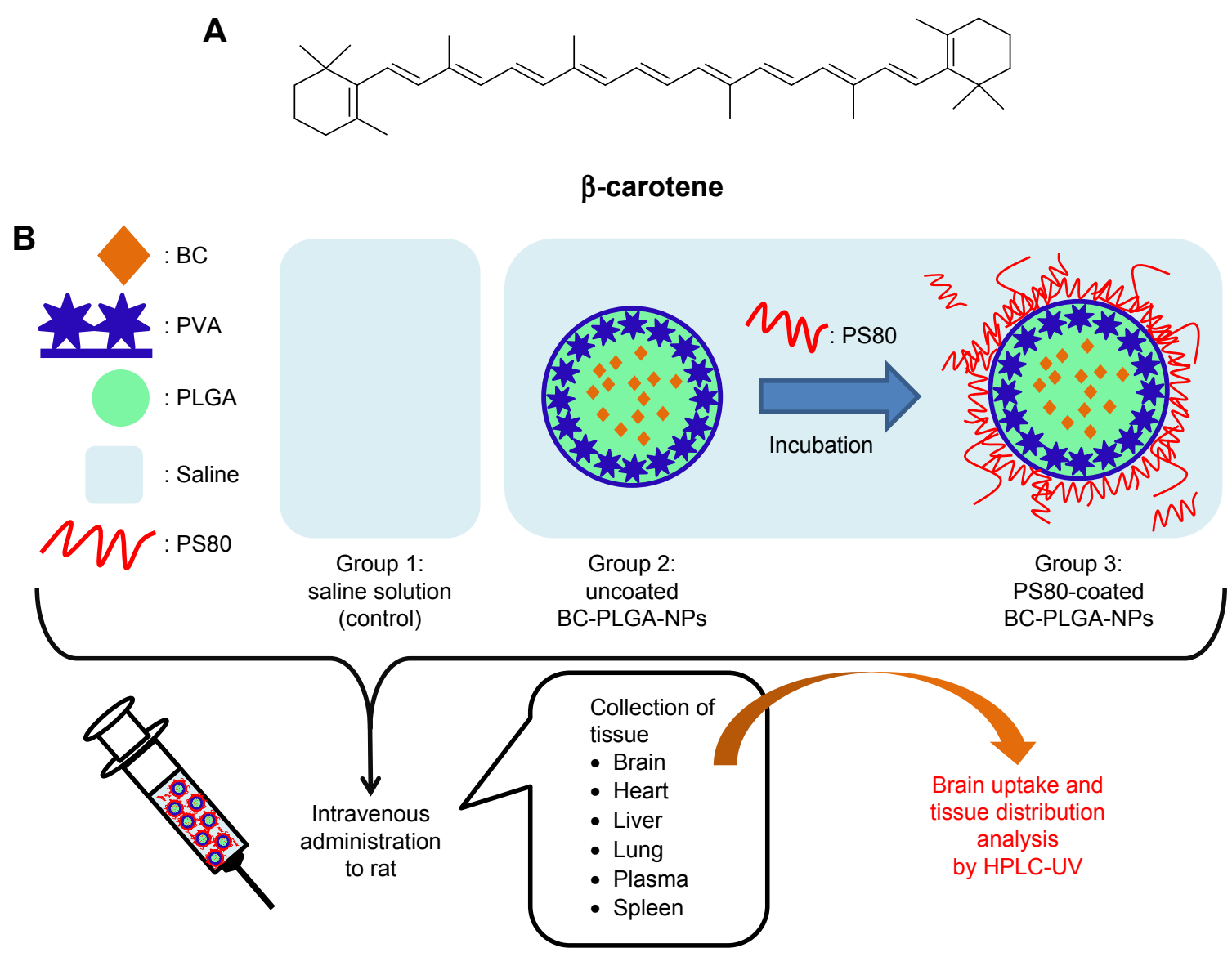

Figure I Chemical structure of $B C$ and experimental design of the present study.

Notes: (A) Chemical structure of naturally occurring BC; (B) schematic experimental design of the present study.

Abbreviations: BC, $\beta$-carotene; BC-PLGA-NPs, $\beta$-carotene-encapsulated poly(D, L-lactide-co-glycolide) nanoparticles; PLGA, poly(D, L-lactide-co-glycolide); PS80, polysorbate 80; PVA, poly vinyl alcohol; HPLC-UV, high performance liquid chromatography with UV detection. 
PLGA were added to $2 \mathrm{~mL}$ of dichloromethane in a $10 \mathrm{~mL}$ glass tube and vortexed for 10 minutes to dissolve them. Next, $4 \mathrm{~mL}$ of $5 \%(\mathrm{w} / \mathrm{v})$ PVA aqueous solution was added and vortexed for 10 seconds. The resultant mixture was sonicated at $40 \%$ amplitude for 3 minutes using a sonic dismembrator, and added dropwise into $100 \mathrm{~mL}$ of $0.3 \%$ (w/v) PVA aqueous solution under rapid stirring. The resultant oil-in-water emulsion was stirred for 9 hours in a fume hood to allow the dichloromethane solvent to evaporate. The suspension was centrifuged at $2,000 \times g$ for 10 minutes at $4^{\circ} \mathrm{C}$ to remove larger aggregates. The supernatant was collected, filtered through a membrane filter, washed three times with Milli-Q water by centrifugation, and finally resuspended in $3 \mathrm{~mL}$ of Milli-Q water either with or without $5 \%(\mathrm{w} / \mathrm{v})$ sucrose. ${ }^{12}$ The resulting BC-PLGA-NP suspension was lyophilized and stored in the dark at $-30^{\circ} \mathrm{C}$. PLGA-NPs were prepared similarly, without addition of $\mathrm{BC}$.

Surface morphology of BC-PLGA-NPs (lyophilized powder) was evaluated by SEM (SU8000 Type II, Hitachi Co., Ltd, Tokyo, Japan). ${ }^{12}$ The effect of sucrose addition on the surface microstructure was also investigated. Lyophilized BC-PLGA-NPs, PLGA-NPs, and free BC powder (100 mg each) were suspended in $3 \mathrm{~mL}$ of distilled water and photographed. To measure $\mathrm{EE}, \mathrm{BC}$ was extracted from the BC-PLGA-NPs by a mixture of ethanol and hexane. ${ }^{9}$ The amount of BC in each extract was determined by HPLC-UV as described in the following section. EE was calculated as follows: $\mathrm{EE}(\%)=\mathrm{BC}_{\text {encapsulated }} / \mathrm{BC}_{\text {total }} \times 100 .{ }^{13}$

\section{Preparation of PS80-coated BC-PLGA- NPs: diameter and zeta potential analysis}

BC-PLGA-NPs (lyophilized powder, $100 \mathrm{mg}$ ) were resuspended in $3 \mathrm{~mL}$ of distilled water containing 1\% PS80, and incubated for 30 minutes to coat the BC-PLGA-NPs with PS80. ${ }^{6,7,14}$ Uncoated BC-PLGA-NPs were prepared similarly without addition of PS80. Uncoated PLGA-NPs were also prepared. All suspensions were subjected to DLS and laser Doppler anemometry measurement using ELS-Z (Otsuka Electronics Co., Ltd., Osaka, Japan) to evaluate the mean diameters and zeta potentials, respectively.

\section{Animal study}

Nine male Sprague Dawley rats (12 weeks of age, 410-580 g body weight) were obtained from CLEA (Central Laboratory for Experimental Animals) Japan Inc. (Tokyo, Japan) and housed in cages kept at $23^{\circ} \mathrm{C}$ with a 12 -hour light:dark cycle. The rats were acclimated with commercial rodent chow (CE-2; CLEA Japan Inc.) and water for 1 week. After acclimatization, rats were fasted for 14 hours, followed by IV administration of $2.4 \mathrm{~mL}$ of PS80-coated BC-PLGA-NPs suspension, uncoated BC-PLGA-NPs suspension, or control saline into the rat tail vein, at a $\mathrm{BC}$ dose of $8.5 \mathrm{mg} / \mathrm{rat}$ (equivalent sum of BC from the three preparations). Rats were sacrificed by decapitation 1 hour after administration, and organs (brain, heart, liver, lungs, and spleen) were harvested. Blood was collected in ethylenediaminetetraacetic acid (EDTA)-coated tubes, and centrifuged at $1,000 \times g$ for 15 minutes at $4^{\circ} \mathrm{C}$ to prepare the plasma samples. Harvested organs and plasma were stored at $-80^{\circ} \mathrm{C}$ until further analysis. The experimental protocol was reviewed by the Committee on the Ethics of Animal Experiments and carried out in accordance with the Animal Experiment Guidelines of Tohoku University. The permit number for this animal experiment is 23-Noudou-23.

BC was extracted from organ samples as follows: ${ }^{15}$ organs were homogenized with saline (containing $1 \mathrm{mM}$ EDTA) to prepare $20 \%(\mathrm{w} / \mathrm{v})$ homogenate solution. The solution $(0.5 \mathrm{~mL})$ was mixed with $2.6 \mathrm{~mL}$ of ethanol (containing $0.9 \mathrm{~mol} / \mathrm{L}$ potassium hydroxide and $0.05 \mathrm{~mol} / \mathrm{L}$ pyrogallol). The mixture was saponified at $80^{\circ} \mathrm{C}$ for 2 hours. After addition of $2 \mathrm{~mL}$ of distilled water, $\mathrm{BC}$ was extracted from the resultant mixture using $3 \mathrm{~mL}$ of hexane/diethyl ether $(3: 1, v / v)$. The extract was partitioned into two layers by centrifugation at $1,000 \times g$ for 10 minutes at $4^{\circ} \mathrm{C}$. The upper hexane layer was collected, and the extraction was repeated. The layers were combined, dried under nitrogen gas, resuspended in a small amount of methanol/methyl tert-butyl ether (MTBE) $(2: 3, \mathrm{v} / \mathrm{v})$, and subjected to BC analysis by HPLC-UV. For plasma BC analysis, plasma $(0.5 \mathrm{~mL})$ was mixed with $0.5 \mathrm{~mL}$ of distilled water, $1 \mathrm{~mL}$ of ethanol, and $3 \mathrm{~mL}$ of hexane. ${ }^{16}$ The mixture was centrifuged at $1,000 \times g$ for 10 minutes at $4^{\circ} \mathrm{C}$. The upper hexane layer was collected, and the extraction was repeated. The layers were combined, dried, resuspended in a small amount of methanol/ MTBE (2:3, v/v), and subjected to analysis by HPLC-UV. These extraction procedures were conducted under subdued (yellow) light to minimize photodegradation of BC.

\section{HPLC-UV determination of BC in sample extract}

Sample extract was analyzed by HPLC-UV. ${ }^{17} \mathrm{~A} \mathrm{C}_{30}$ carotenoid column (4.6×250 mm, $5 \mu \mathrm{m}$; YMC, Kyoto, Japan) was used, and the extract was eluted using a binary gradient consisting of the following HPLC solvents: A, methanol/MTBE/ water $(83: 15: 2[\mathrm{v} / \mathrm{v} / \mathrm{v}]$, containing $3.9 \mathrm{mmol} / \mathrm{L}$ ammonium acetate); B, methanol/MTBE/water $(8: 90: 2[\mathrm{v} / \mathrm{v} / \mathrm{v}]$, containing $2.6 \mathrm{mmol} / \mathrm{L}$ ammonium acetate). The gradient profile was 
as follows: B linear ratio; $10 \%-55 \%$ at $0-12$ minutes, $55 \%-100 \%$ at $12-20$ minutes, $100 \%$ at $20-25$ minutes, and $100 \%-10 \%$ at $25-27$ minutes. The flow rate was adjusted to $1 \mathrm{~mL} / \mathrm{min}$, and the column temperature was maintained at $30^{\circ} \mathrm{C}$. The column eluent was sent to a UV detector (UV-2075 PLUS, JASCO, Tokyo, Japan) for BC monitoring at $463 \mathrm{~nm}$. Concentrations of $\mathrm{BC}$ were calculated using the equation corresponding to the standard curve.

\section{Statistical analysis}

Results are reported as mean $\pm \mathrm{SD}$. Statistical significance between the PS80-coated and uncoated BC-PLGA-NP groups was analyzed using the independent Student's $t$-test. Mean significantly differed at $P<0.05$ and $P<0.01$.

\section{Results}

\section{Surface morphology and EE analysis of BC-PLGA-NPs}

BC-PLGA-NPs were prepared by solvent evaporation, and suspended in water in either the presence or absence of a cryoprotectant (sucrose). SEM images of the BC-PLGA-NP suspension lyophilized in the absence of sucrose revealed spherical aggregates (Figure 2A). Lyophilization in the presence of sucrose, on the other hand, resulted in the BCPLGA-NPs being immobilized in the sucrose matrix, with no evidence of aggregation (Figure 2B). We therefore used sucrose for the preparation of lyophilized BC-PLGA-NPs in this study.

Dispersion of lyophilized powder of PLGA-NPs in distilled water resulted in a transparent homogeneous suspension with white color (Figure 3A). In contrast, dispersion of BC-PLGA-NPs resulted in orange homogeneous suspension
(Figure 3B). The EE of BC in the BC-PLGA-NPs was estimated to be $14.2 \% \pm 0.3 \%(n=3$, Table 1$)$. Free BC powder was very difficult to disperse and showed a deep red color (Figure 3C).

\section{Mean diameter and zeta potential analysis of PS80-coated BC-PLGA-NPs}

BC-PLGA-NPs were coated with PS80 by incubation in water containing 1\% PS80. Mean diameters and zeta potentials of all preparations were nearly identical (Table 1). Mean diameters of PS80-coated BC-PLGA-NPs, uncoated BC-PLGA-NPs, and uncoated PLGA-NPs were found to be $259 \pm 62 \mathrm{~nm}, 286 \pm 93 \mathrm{~nm}$, and $256 \pm 25 \mathrm{~nm}(\mathrm{n}=3)$, respectively. Zeta potentials of each of the preparations were $-26.36 \pm 0.98 \mathrm{mV},-29.92 \pm 3.25 \mathrm{mV}$, and $-29.31 \pm 2.73 \mathrm{mV}$ $(\mathrm{n}=3)$, respectively. The resultant PS80-coated and uncoated BC-PLGA-NPs were employed to evaluate the brain uptake and tissue distribution of $\mathrm{BC}$ in rats.

\section{Brain uptake and tissue distribution of $B C$ following IV administration of PS80-coated BC-PLGA-NPs}

Rats were intravenously administered with suspensions of PS80-coated or uncoated BC-PLGA-NPs, or saline control. Before IV administration, we did not reduce excess PS80 in the solution because several reports showed brain uptake of PS80-coated NPs with this manner..$^{5-7,14}$ One hour after administration, blood plasma was collected and organs were harvested, and the corresponding concentrations of BC were determined by HPLC-UV (Figure 4). No detectable levels of $\mathrm{BC}$ were found in the plasma or any of the organs of rats that received saline (control).
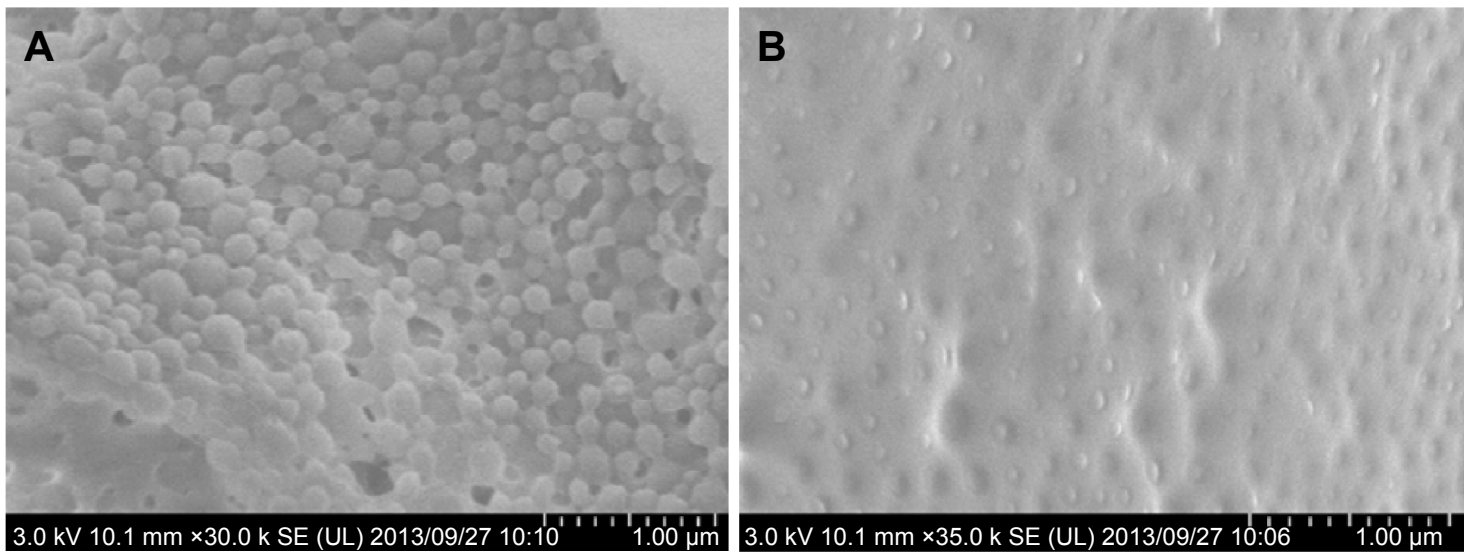

Figure 2 SEM images of lyophilized BC-PLGA-NPs in the presence and absence of $5 \%(w / v)$ sucrose.

Notes: (A) Lyophilized BC-PLGA-NPs in the absence of sucrose. (B) Lyophilized BC-PLGA-NPs in the presence of $5 \%$ (w/v) sucrose.

Abbreviations: BC-PLGA-NPs, $\beta$-carotene-encapsulated poly(D, L-lactide-co-glycolide) nanoparticles; SEM, scanning electron microscope. 


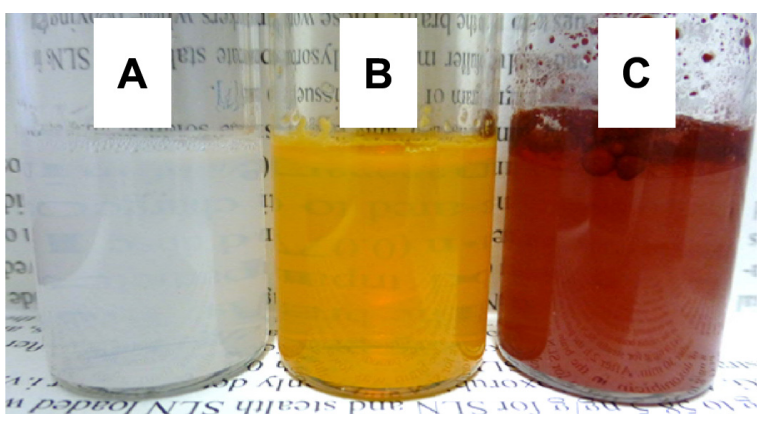

Figure 3 Photographs of NPs and control suspensions.

Notes: Lyophilized products were redispersed in distilled water and mixed for 5 minutes, and then photographed. (A): PLGA-NPs lyophilized with 5\% (w/v) sucrose. (B): BC-PLGA-NPs lyophilized with $5 \%(w / v)$ sucrose. (C): Free BC powder in distilled water.

Abbreviations: $B C, \beta$-carotene; $B C-P L G A-N P s, \beta$-carotene-encapsulated poly $(D$, L-lactide-co-glycolide) nanoparticles; NPs, nanoparticles; PLGA-NPs, poly(D, L-lactide-co-glycolide) nanoparticles.

In contrast, no significant differences of brain $\mathrm{BC}$ concentration were found between BC-PLGA-NP and PS80-coated BC-PLGA-NP groups. These unexpected findings suggest that coating with PS80 did not have an impact on BC brain uptake. On the other hand, concentrations of $\mathrm{BC}$ in the lungs and plasma were significantly higher and lower, respectively, in the PS80-coated BC-PLGA-NP group, compared with the uncoated BC-PLGA-NP group. Increased levels of $\mathrm{BC}$ were also found in the heart, liver, and spleen in the PS80-coated BC-PLGA-NP group, compared with the uncoated group. Taken together, these results suggest that PS80-coated BC-PLGA-NPs were quickly transferred from the circulation to the lungs, rather than to the brain, while uncoated BC-PLGA-NPs were not distributed to the lungs and remained in circulation.

\section{Discussion}

BC (Figure 1A) exhibits antioxidative properties and plays an important role in protecting cellular membrane lipids from

Table I Physicochemical parameters of the PS80-coated BCPLGA-NPs, uncoated BC-PLGA-NPs, and uncoated PLGA-NPs

\begin{tabular}{|c|c|c|c|}
\hline Groups & $\begin{array}{l}\text { Mean diameter } \\
(\mathrm{nm})\end{array}$ & $\begin{array}{l}\text { Zeta potential } \\
(\mathrm{mV})\end{array}$ & $\begin{array}{l}\text { Encapsulation } \\
\text { efficiency (\%) }\end{array}$ \\
\hline $\begin{array}{l}\text { PS80-coated } \\
\text { BC-PLGA-NPs }\end{array}$ & $259 \pm 62$ & $-26.36 \pm 0.98$ & $14.2 \pm 0.3$ \\
\hline $\begin{array}{l}\text { Uncoated } \\
\text { BC-PLGA-NPs }\end{array}$ & $286 \pm 93$ & $-29.92 \pm 3.25$ & \\
\hline $\begin{array}{l}\text { Uncoated } \\
\text { PLGA-NPs }\end{array}$ & $256 \pm 25$ & $-29.3 \mathrm{I} \pm 2.73$ & \\
\hline
\end{tabular}

Note: Values represent mean $\pm S D, n=3$.

Abbreviations: BC-PLGA-NPs, $\beta$-carotene-encapsulated poly(D, L-lactide-coglycolide) nanoparticles; PLGA-NPs, poly(D, L-lactide-co-glycolide) nanoparticles; PS80, polysorbate 80 . oxidative stress in the body. ${ }^{18}$ Recently, encapsulation of BC in PLGA-NPs has been carried out in an effort to improve its therapeutic effects. For example, Yusuf et al prepared PS80coated BC-PLGA-NPs and found that IP administration in rats significantly increased $\mathrm{BC}$ concentrations in the brain. ${ }^{9}$ However, they did not evaluate the distribution of $\mathrm{BC}$ into other organs. Elucidating the whole-body distribution of BC is important to gauge the usefulness of encapsulation methods and PS80 coating. To clarify these aspects, we prepared PS80-coated BC-PLGA-NPs and uncoated BC-PLGA-NPs, and investigated the brain uptake and tissue distribution profiles of BC following IV administration in rats.

BC-PLGA-NPs were lyophilized in the presence of sucrose, and the resultant BC-PLGA-NPs were immobilized (protected) in the sucrose matrix (Figure 2B). This is consistent with results of a previous study ${ }^{12}$ in which SEM investigation of the surface morphology of PLGA-NPs revealed the protective effects of sucrose during the lyophilization process. In this study, the lyophilized powder (BC-PLGA-NPs) was well-dispersed in water, with no apparent aggregation (Figure 3B). Thus, lyophilization of BC-PLGA-NPs in the presence of sucrose is recommended to obtain well-dispersed BC-PLGA-NPs.

The EE of drugs in PLGA-NPs is generally reported to be approximately $45 \% \cdot{ }^{13}$ In the present study, the EE of BC was found to be rather low (14.2\%), which may be due to aggregation of the BC-PLGA-NPs during preparation. Specifically, we prepared BC-PLGA-NPs by solvent evaporation. During the solvent (dichloromethane) evaporation process, visible aggregates of $\mathrm{BC}$ were observed. Additional studies are needed to focus on optimizing the amounts of PLGA and PVA to overcome this problem.

Coating with PS80 did not impact the mean diameter and zeta potential of NPs. Specifically, the mean diameter (286 $\mathrm{nm})$ and zeta potential $(-29.9 \mathrm{mV})$ of the BC-PLGA-NPs were found to be similar to those of general PLGA-NPs prepared by solvent evaporation. ${ }^{5-7,13}$ As a point of reference, Ribeiro et al prepared polysorbate 20 (PS20)-coated BC-PLGA-NPs by solvent evaporation of an oil-in-water emulsion comprising PS20. ${ }^{19}$ Using this same procedure, Yusuf et al prepared PS80-coated BC-PLGA-NPs. ${ }^{9}$ While these methods are useful for surface coating, it is nearly impossible to produce uncoated BC-PLGA-NPs, so that comparisons between PS80-coated and uncoated BC-PLGANPs cannot be performed. In this study, we first prepared uncoated BC-PLGA-NPs, and then incubated the resulting BC-PLGA-NPs with PS80 to allow for surface coating. ${ }^{6,7,14}$ Both coated and uncoated BC-PLGA-NPs were administered 

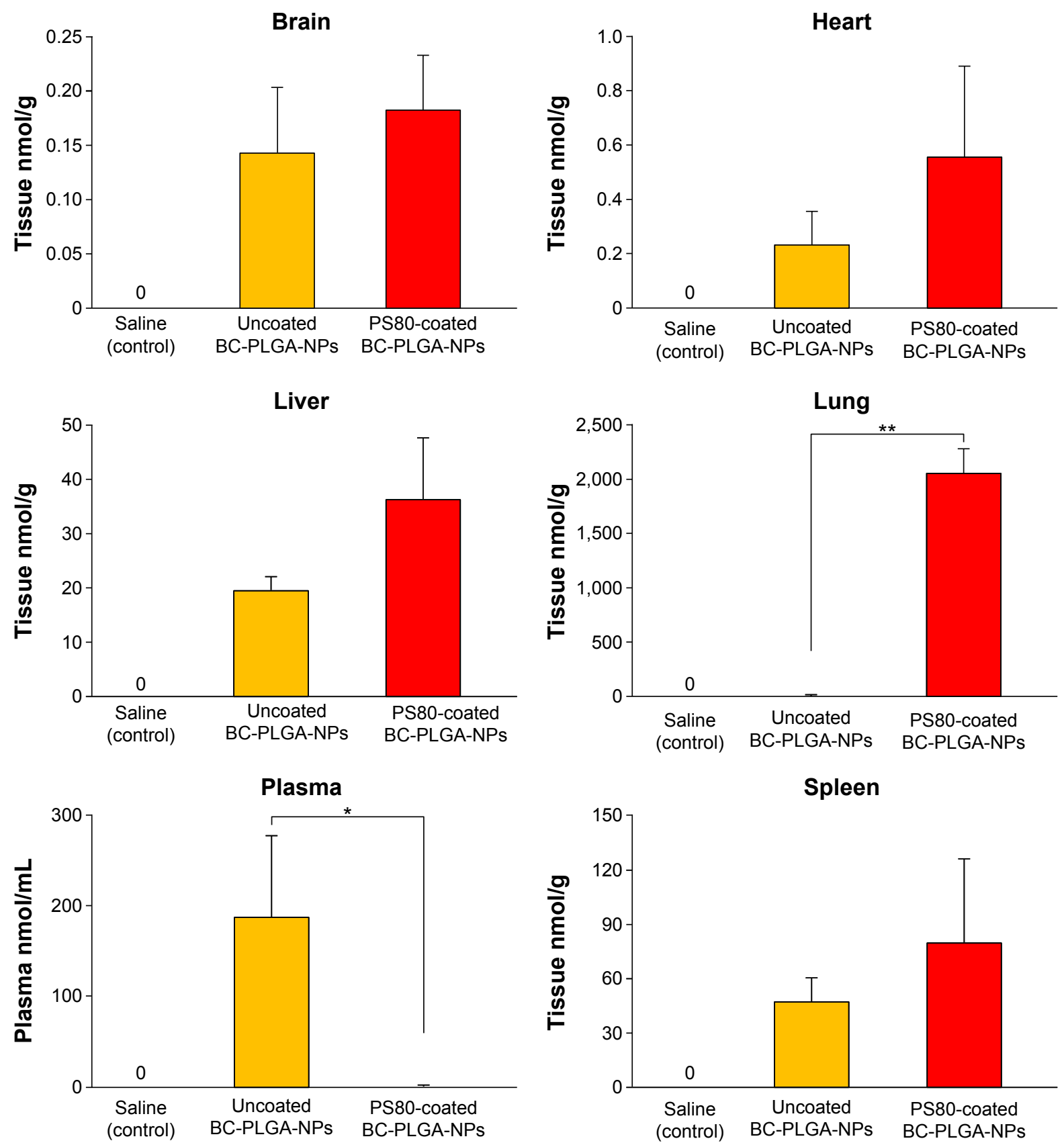

Figure 4 BC concentrations in the organs and plasma of rats after IV administration of saline control, uncoated BC-PLGA-NPs, and PS80-coated BC-PLGA-NPs.

Notes: $B C$ concentrations in organs and plasma were analyzed I hour after IV administration (mean $\pm S D, n=3$ ). Data were calculated using the value given by the independent Student's $t$-test between the PS80-coated and uncoated BC-PLGA-NP groups. Mean significantly differed at $* P<0.05$ and $* * P<0.01$.

Abbreviations: BC, $\beta$-carotene; BC-PLGA-NPs, $\beta$-carotene-encapsulated poly(D, L-lactide-co-glycolide) nanoparticles; PS80, polysorbate 80; IV, intravenous.

intravenously to rats, allowing for a direct comparison of their distribution profiles in various tissues.

Generally, rodents exhibit very low tissue BC concentrations in the body. ${ }^{20}$ Consistent with this, no detectable levels of $\mathrm{BC}$ were found in the brain and other tissues in the control group (Figure 4). Blood plasma was collected and organs were harvested 1 hour after administration, since previous in vivo studies found higher brain uptake of PS80coated PLGA-NPs at this time point. ${ }^{5,7}$ In this study, however, the PS80-coated BC-PLGA-NP group did not result in higher concentrations of $\mathrm{BC}$ in the brain (Figure 4, brain). So far, only two published studies ${ }^{5,6}$ have compared PS80-coated and uncoated PLGA-NPs having similar mean diameters (approximately $250 \mathrm{~nm}$ ) to the NPs used herein. Both studies, indeed, revealed increased brain uptake of PS80-coated PLGA-NPs. However, mannitol was used as the cryoprotectant for the preparation of the NPs in these studies. As mannitol is known as a transient blood-brain barrier opener, ${ }^{21}$ 
further studies are needed to clarify the effect of mannitol on the brain uptake of PS80-coated BC-PLGA-NPs.

Plasma BC concentrations in the PS80-coated BCPLGA-NP group were found to be much lower than those in the uncoated BC-PLGA-NP group (Figure 4, plasma). Such prolonged circulation effect of uncoated BC-PLGA-NPs was reported by Tsai et $\mathrm{al}^{13}$ using curcumin-encapsulated uncoated PLGA-NPs (approximately $160 \mathrm{~nm}$ in diameter). Considering the release profile, Capurso et $\mathrm{al}^{10}$ prepared retinoic acid-encapsulated PLGA-NPs (approximately $250 \mathrm{~nm}$ in diameter) with a similar procedure to us. They found $20 \%$ of retinoic acid was released from PLGA-NPs within 10 hours of incubation in phosphate-buffered saline. Therefore, in the present study, we deduced that approximately $20 \%$ of BC from BC-PLGA-NPs may be released. But, almost all of BC were still encapsulated in NPs within 1 hour. On the other hand, the PS80-coated BC-PLGA-NP group tended to result in higher $\mathrm{BC}$ accumulation in other organs (Figure 4, heart, liver, and spleen). Interestingly, $\mathrm{BC}$ concentrations in the lungs in the PS80-coated BC-PLGA-NP group were much higher than those in the uncoated BC-PLGA-NP group (Figure 4, lung). Löbenberg et al reported that PS80-coated hexyl-cyanoacrylate (encapsulating azidothymidine) NPs (approximately $230 \mathrm{~nm}$ in diameter) exhibited a similar tissue distribution profile. ${ }^{22}$ In general, microparticles (larger than $7 \mu \mathrm{m}$ ) are known to significantly accumulate in the lungs following IV administration. ${ }^{23}$ As the PS80-coated BCPLGA-NPs used in the present study were on the nanoscale (approximately $260 \mathrm{~nm}$ in diameter), it is difficult to speculate that their sizes could affect BC accumulation in the lungs. Considering together the results of previous studies ${ }^{4,24}$ and the present findings, both the accumulation of $\mathrm{BC}$ in the lungs and the unique tissue distribution observed for BC (Figure 4) may be explained by distinct binding of apolipoproteins (eg, apolipoprotein E) to the surface of PS80-coated or uncoated BC-PLGA-NPs in circulation. Coating with PS80 may induce the binding of certain apolipoproteins to the surface of BC-PLGA-NPs, thereby enhancing their uptake into macrophage-rich organs, such as the lungs. Further investigations of longer time study (such as 6 or 12 hours) are needed to clarify these phenomenon. Moreover, Pradeep and Kuttan reported that IP administration of BC suspended in gum acacia solution inhibited lung metastasis in mice, ${ }^{25}$ suggesting that our results (ie, improvement of $\mathrm{BC}$ levels in the lungs using PS80-coated BC-PLGA-NPs) may have healthrelated applications. Also, our finding would enable the use of other beneficial carotenoids such as $\alpha$-carotene, astaxanthin, $\beta$-cryptoxanthin, lutein, lycopene, and neoxanthin.

\section{Conclusion}

BC-PLGA-NPs were successfully prepared by solvent evaporation. Addition of sucrose was found to prevent aggregation of the BC-PLGA-NPs during the lyophilization process. PS80-coated BC-PLGA-NPs resulted in significant accumulation of BC in the lungs 1 hour after IV administration, suggesting that PS80-coated BC-PLGA-NPs are quickly transferred from the circulation to the lungs. Uncoated BCPLGA-NPs, on the other hand, revealed prolonged plasma circulation. Taken together, these results suggest that coating the surface of BC-PLGA-NPs with PS80 can significantly change the entire tissue distribution profile. Further, increased accumulation of BC in the lungs may be useful for the treatment of pulmonary disease.

\section{Acknowledgment}

This work was supported in part by KAKENHI (15K14725) of the Japan Society for the Promotion of Science, Japan.

\section{Disclosure}

Taiki Miyazawa was supported by Japan Society for the Promotion of Science Postdoctoral Fellowships for Research Abroad (http://www.jsps.go.jp/). The authors report no other conflicts of interest in this work.

\section{References}

1. Alexis F, Pridgen E, Molnar LK, Farokhzad OC. Factors affecting the clearance and biodistribution of polymeric nanoparticles. Mol Pharm. 2008;5(4):505-515.

2. Danhier F, Ansorena E, Silva JM, Coco R, Le Breton A, Préat V. PLGAbased nanoparticles: an overview of biomedical applications. $J$ Control Release. 2012;161(2):505-522.

3. Kumari A, Yadav SK, Yadav SC. Biodegradable polymeric nanoparticles based drug delivery systems. Colloids Surf B Bioint. 2010; 75(1):1-18.

4. Aggarwal P, Hall JB, McLeland CB, Dobrovolskaia MA, McNeil SE. Nanoparticle interaction with plasma proteins as it relates to particle biodistribution, biocompatibility and therapeutic efficacy. Adv Drug Deliv Rev. 2009;61(6):428-437.

5. Tahara K, Miyazaki Y, Kawashima Y, Kreuter J, Yamamoto H. Brain targeting with surface-modified poly(D,L-lactic-co-glycolic acid) nanoparticles delivered via carotid artery administration. Eur J Pharm Biopharm. 2011; $77(1): 84-88$.

6. Gelperina S, Maksimenko O, Khalansky A, et al. Drug delivery to the brain using surfactant-coated poly (lactide-co-glycolide) nanoparticles: influence of the formulation parameters. Eur J Pharm Biopharm. 2009; 74(2):157-163.

7. Chaturvedi M, Molino Y, Sreedhar B, Khrestchatisky M, Kaczmarek L. Tissue inhibitor of matrix metalloproteinases-1 loaded poly(lactic-coglycolic acid) nanoparticles for delivery across the blood-brain barrier. Int J Nanomedicine. 2014;9:575-588.

8. Gonnet M, Lethuaut L, Boury F. New trends in encapsulation of liposoluble vitamins. J Control Release. 2010;146(3):276-290.

9. Yusuf M, Khan RA, Khan M, Ahmed B. Plausible antioxidant biomechanics and anticonvulsant pharmacological activity of braintargeted $\beta$-carotene nanoparticles. Int J Nanomedicine. 2012;7: 4311-4321. 
10. Capurso NA, Look M, Jeanbart L, et al. Development of a nanoparticulate formulation of retinoic acid that suppresses Th17 cells and upregulates regulatory T cells. Self Nonself. 2010;1(4):335-340.

11. Murakami H, Kobayashi M, Takeuchi H, Kawashima Y. Preparation of poly(DL-lactide-co-glycolide) nanoparticles by modified spontaneous emulsification solvent diffusion. Int J Pharm. 1999;187(2): $143-152$.

12. Tang KS, Hashmi SM, Shapiro EM. The effect of cryoprotection on the use of PLGA encapsulated iron oxide nanoparticles for magnetic cell labeling. Nanotechnology. 2013;24(12):125101.

13. Tsai YM, Chien CF, Lin LC, Tsai TH. Curcumin and its nanoformulation: the kinetics of tissue distribution and blood-brain barrier penetration. Int J Pharm. 2011;416(1):331-338.

14. Wohlfart S, Khalansky AS, Gelperina S, Begley D, Kreuter J. Kinetics of transport of doxorubicin bound to nanoparticles across the bloodbrain barrier. J Control Release. 2011;154(1):103-107.

15. Lunetta JM, Zulim RA, Dueker SR, et al. Method for the simultaneous determination of retinol and beta-carotene concentrations in human tissues and plasma. Anal Biochem. 2002;304(1):100-109.

16. Lyan B, Azais-Braesco V, Cardinault N, et al. Simple method for clinical determination of 13 carotenoids in human plasma using an isocratic high-performance liquid chromatographic method. J Chromatogr B Biomed Sci Appl. 2001;751(2):297-303.

17. Miyazawa T, Nakagawa K, Miyazawa T. Liquid chromatography-based assay for carotenoids in human blood. In: Preedy VR, editor. Vitamin A and Carotenoids: Chemistry, Analysis, Function and Effects, Series of Food and Nutritional Compounds in Focus. Cambridge, UK: Royal Society of Chemistry; 2012:184-203 (ISBN: 9781849733687).
18. Nakagawa K, Fujimoto K, Miyazawa T. $\beta$-Carotene as a high-potency antioxidant to prevent the formation of phospholipid hydroperoxides in red blood cells of mice. Biochim Biophys Acta. 1996;1299(1):110-116.

19. Ribeiro HS, Chu BS, Ichikawa S, Nakajima M. Preparation of nanodispersions containing $\beta$-carotene by solvent displacement method. Food Hydrocolloids. 2008;22(1):12-17.

20. Ribaya-Mercado JD, Holmgren SC, Fox JG, Russell RM. Dietary beta-carotene absorption and metabolism in ferrets and rats. J Nutr. 1989;119(4):665-668.

21. Brown RC, Egleton RD, Davis TP. Mannitol opening of the blood-brain barrier: regional variation in the permeability of sucrose, but not $86 \mathrm{Rb}+$ or albumin. Brain Res. 2004;1014(1-2):221-227.

22. Löbenberg R, Araujo L, Briesen HV, Rodgers E, Kreuter J. Body distribution of azidothymidine bound to hexyl-cyanoacrylate nanoparticles after i.v. injection to rats. J Control Release. 1998;50(1-3):21-30.

23. Azarmi S, Roa WH, Löbenberg R. Targeted delivery of nanoparticles for the treatment of lung diseases. Adv Drug Deliv Rev. 2008;60(8): 863-875.

24. Sempf K, Arrey T, Gelperina S, et al. Adsorption of plasma proteins on uncoated PLGA nanoparticles. Eur J Pharm Biopharm. 2013;85(1): 53-60.

25. Pradeep CR, Kuttan GE. Effect of $\beta$-Carotene on the inhibition of lung metastasis in mice. Phytomedicine. 2003;10(2-3):159-164.
International Journal of Nanomedicine

\section{Publish your work in this journal}

The International Journal of Nanomedicine is an international, peerreviewed journal focusing on the application of nanotechnology in diagnostics, therapeutics, and drug delivery systems throughout the biomedical field. This journal is indexed on PubMed Central, MedLine, CAS, SciSearch $®$, Current Contents ${ }^{\circledR} /$ Clinical Medicine,

\section{Dovepress}

Journal Citation Reports/Science Edition, EMBase, Scopus and the Elsevier Bibliographic databases. The manuscript management system is completely online and includes a very quick and fair peer-review system, which is all easy to use. Visit http://www.dovepress.com/ testimonials.php to read real quotes from published authors. 